\title{
Correspondence
}

Epidemiol. Infect. (2013).

doi: $10.1017 /$ S0950268812003068

First published online 18 January 2013

\section{What can the 2009 influenza outbreak teach us about the risk of a severe pandemic? The Madagascar experience}

To the Editor:

The recent work of Rajatonirina and collaborators [1], from the Pasteur Institute in Madagascar, represents an important contribution to the understanding of mortality associated with the so-called 2009 influenza pandemic through the analysis of mortality rates and viral surveillance data from Antananarivo. This is the capital of a country within a continent of great climatic and social diversity, yet where data on the 2009 influenza outbreak are extremely sparse [2]. The authors found that during the peak in the detection of positive specimens for the $\mathrm{H} 1 \mathrm{~N} 1 \mathrm{pdm}$ strain in November 2009, 'There were $20 \%$ more human deaths than expected' compared to the previous two years. The authors therefore conclude that such findings 'are consistent with the notion that African populations may be at higher risk of death from pandemic influenza'. Here we explain why we are sceptical about the claim of high severity of this strain in Madagascar, particularly in light of inherent limitations associated with the data available. More importantly, we argue that attempting to infer the risks of a future pandemic based on the 2009 experience can be misleading, as the impact of the H1N1pdm strain resembled more that of a (severe) seasonal influenza strain than that of a pandemic [3].

The declaration of a pandemic in June 2009 by the World Health Organization was not free of controversy $[3,4]$, because by the time of the declaration it was already clear that this strain was not as severe as first feared. Still, the pandemic label became attached to this strain, with various subsequent studies performed to determine its impact. We have performed one such study, in which we estimated the anomalous burden of influenza throughout Brazil during 2009 [5]. Unexpectedly, we found no substantial excess of deaths above the usual annual variance in mortality in the equatorial region of the country, in contrast with the higher mortality rates in adults in the temperate southern states.

Therefore coming from the Brazilian experience, it is fair to disclose, we have a more enquiring approach to claims of a high H1N1pdm burden in other equatorial regions. Following inspection of the data from Antananarivo, we identified four major problems with the notion of an unusually high mortality associated with the circulation of the H1N1strain in 2009. First, the analysis of the data from Antananarivo shows that when mortality rates are considered (as the population growth in that city is nearly $5 \%$ per year), the only significant increase in mortality in November 2009 was in the elderly population. This is in sharp contrast to typical findings of pandemic-associated mortality in the young adult population (although we concede that this could be credited to an interesting local exception). Second, there was already a trend towards higher mortality rates only in this same age group from 2007 to 2008, well before the circulation of the pandemic strain. Such a trend was present when all months, as well as November alone, were examined (Fig. 2 of Rajatonirina et al. [1]). Third, the relevance of the observed increase in mortality in November should also be viewed with caution if we consider, as the authors point out, that the number of deaths was probably underreported in the first months of 2009 (the period associated with the highest peaks of mortality in previous years) due to the civil crisis in Madagascar, thereby masking a potentially flatter mortality pattern throughout 2009. Last, and perhaps most importantly, it should also be noted that even if the observed increment of mortality in 
November had been caused predominantly by the H1N1pdm strain, observation of the entire mortality series in that region (from 2007 to 2009) revealed similarly high peaks of mortality in previous years. For instance, mortality at week 4 of 2008 was as high as the supposedly pandemic peak in November 2009, even though the population in 2008 was smaller. Therefore, the reported increment of deaths in November 2009 was not particularly anomalous considering the usual background variance of mortality in Antananarivo.

A recently released compilation of studies on influenza in Africa [2] describes a wealth of interesting data from this continent. Unfortunately, these studies were not supplemented with time series of mortality from vital statistics sources that would have allowed comparison of the anomalous mortality attributable to $\mathrm{H} 1 \mathrm{~N} 1 \mathrm{pdm}$ relative to previous years. Still, the data presented are overly indicative that the introduction of the H1N1pdm strain was not followed by a sharp rise in cases and deaths in the tropical belt. For instance, the delayed timing of the circulation of this strain in West Africa and its impact, comparable to inter-pandemic periods [6], is markedly similar to that described for equatorial Brazil [5].

If further analyses do not reveal an exceptionally high number of deaths in Madagascar and other tropical African countries during the circulation of H1N1pdm, should we instead conclude that African populations are at lower risk from pandemic influenza? The answer is 'no'. Independently of the controversy regarding the merits of the criteria used to define this strain as pandemic [2,3], we believe that the 2009 experience is of poor value to predict incidence patterns, transmissibility and burden of a severe pandemic in the future. In fact, by strongly focusing on the pandemic categorization of this H1N1 strain, we might be actually missing the opportunity to understand the dynamics and impact of seasonal influenza based on the unprecedented surveillance efforts that the $2009 \mathrm{H} 1 \mathrm{~N} 1$ experience provided. Therefore, although the study in Antananarivo, and similar studies in Africa, are undoubtedly of great importance to understand the dynamics of influenza in these poorly studied areas, to get a picture of whether African populations might be at higher risk in a putative severe influenza outbreak in the future, one would still have to look at the impact of the 1918 pandemic [7] (and in fact act quickly before this information is irreversibly lost [8]). This is still the closest reference we have of an epidemiological catastrophe of global proportions caused by a respiratory disease in modern times.

\section{Declaration of Interest}

None.

\section{References}

1. Rajatonirina $\mathbf{S}$, et al. Excess mortality associated with the 2009 A(H1N1)v influenza pandemic in Antananarivo, Madagascar. Epidemiology \& Infection. Published online: 20 July 2012. doi:10.1017/S0950268812001215.

2. Katz MA, et al. Influenza in Africa: uncovering the epidemiology of a long-overlooked disease. Journal of Infectious Diseases 2012; 206: S1-S4.

3. Doshi P. The elusive definition of pandemic influenza. Bulletin of the World Health Organization 2011; 89: $532-538$.

4. Morens DM, Folkers GK, Fauce AS. What is a pandemic? Journal of Infectious Diseases 2009; 200: 1018-1021.

5. Schuck-Paim C, et al. Were equatorial regions less affected by the 2009 influenza pandemic? The Brazilian experience. PLoS ONE 2012; 7: e41918.

6. Nzussouo NT, et al. Delayed 2009 pandemic influenza A virus subtype H1N1 circulation in West Africa, May 2009-April 2010. Journal of Infectious Diseases 2012; 206: S101-S107.

7. Murray C, et al. Estimation of potential global pandemic influenza mortality on the basis of vital registry data from the 1918-20 pandemic: a quantitative analysis. Lancet 2006 ; 368 : 2211-2218.

8. Alonso WJ, et al. The fate of historical death certificates: the silent burning of another library of Alexandria. American Journal of Public Health 2012; 102: e1-2.

W. J. ALONSO ${ }^{1,2}$, C. SCHUCK-PAIM ${ }^{2}$

${ }^{1}$ Fogarty International Center, National Institutes of Health (NIH), Bethesda, USA

${ }^{2}$ Origem Scientifica, Sao Paulo, Brazil

Author for correspondence:

W. J. Alonso

Fogarty International Center,

National Institutes of Health (NIH),

16 Center Drive, Building 16, 20892,

Bethesda, MD, USA

(Email: alonsow@mail.nih.gov)

Epidemiol. Infect. (2013).

doi:10.1017/S095026881200307X

First published online 18 January 2013

\section{What can the 2009 influenza outbreak teach us about the risk of a severe pandemic? The Madagascar experience: a reply}

It is well-established that the prevalence and incidence of influenza in most tropical countries like Madagascar are largely unknown. Clinically, 